\title{
sciendo BULK MODULUS OF COPPER ALLOYS IN CONTEXT OF MODERN METAL THEORY
}

DOI 10.2478/ntpe-2018-0086

\author{
Dr. Janusz Chrzanowski \\ Dr. Bohdan Bieg \\ Maritime University of Szczecin, Poland
}

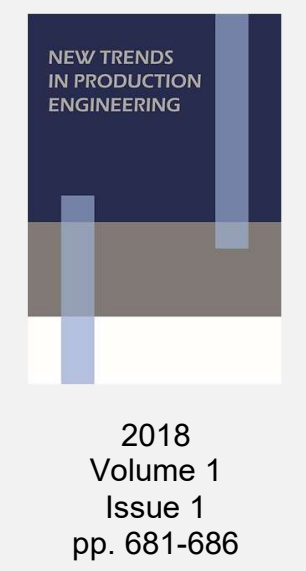

\begin{abstract}
The authors used a modern quantum theory allowing to determine the energy levels of electrons in real metal. Based on that analytical equations have been presented by means of which the bulk modulus for chosen metals were calculated. It should be emphasized that all values obtained directly from the derived equation are in perfect conformity with the experimental data, a few percentage differences are comparable with the potential measurement error. Subsequently a simple mathematical model has been proposed which allows to calculate the bulk modulus of copper alloys depending on their percentage composition. The authors performed numerical calculations for the typical copper alloys and the results are presented in the form of graphs.
\end{abstract}

Keywords: convolution, nonlocal potential, ionization energy, copper alloys

\section{INTRODUCTION}

The high corrosion resistance, strength, and low life-cycle cost of cast-copper alloys make them economical choices for pipes, flanges and fittings, pumps and valve components, bearings and other mechanical devices used in in the shipbuilding industry. In piping, the antifouling characteristics of the copper alloys reduce maintenance costs considerably compared with galvanized steel, rubber-lined products, or fiberglass-reinforced plastic, all of which may be cheaper initially. Also the extraordinarily complex electrical equipment in a ship (the generators, electric motors, lighting and communications systems) depend almost entirely upon copper and copper alloys for their operation.

The shipbuilding industry has sponsored extensive research and development directed the use of copper-nickel hull materials for commercial vessels and for offshore structures. The idea of cladding boats with copper metals has been utilized since the $17^{\text {th }}$ century; copper plates were commonly used in the to protect wooden hulls against wood-boring ship worms. Because early copper-clad craft were also free from the fronds of algae that formed on wooden hulls, they were significantly faster than unclad vessels. In the case of offshore structures, the presence of fouling requires the use of a heavy structure to resist the wave action on the increased cross section presented by the contaminated surface. The study has shown that for large cargo ships, tankers, and naval vessels, thin plates of copper-nickel fitted over conventional steel hulls offer the same advantages as solid or clad copper-alloy plate at significantly lower cost (Powell and Webster, 2012). It should also be emphasized that copper and its alloys are fully recyclable without loss of properties. Globally, $34 \%$ ( $45 \%$ in Europe) of the copper used in new alloys comes from recycled scrap. This provides benefits in energy saving and preservation of resources which contribute to the sustainable qualifications of copper and its alloys.

In presented paper the authors use modern quantum theory to design single-electron states in the real metal (Chrzanowski, 2013). Based on that, analytical equations have been presented by means of which the bulk modulus for copper and chosen metals were calculated. Subsequently a simple mathematical model has been proposed which allow to calculate the bulk modulus of copper alloys depending on their percentage composition. The structure of the article looks as follows; the first chapter presents the foundations of new quantum theory. Next chapter 
introduces the analytical mathematical equations for the bulk modulus of metal. Then these equations are used to calculate the bulk modulus and to compare the obtained results with the experimental data for copper and selected metals. Subsequently, simple mathematical models are presented that allow to calculate how the bulk modulus depends on the percentage composition of selected copper alloys. The last chapter contains a summary and conclusions.

\section{CONVOLUTION POTENTIAL OPERATOR}

The elementary problem in metals theory which has to be solved is how to deal mathematically with the interaction of a large number of particles. In the general case, the full Hamiltonian should contain complete information about the Coulomb interaction between all electrons and ions of the system. It is obvious that all efforts to find the eigenvalues and the eigenfunctions to this Hamiltonian involve approximations (Ashcroft and Mermin, 1976, Harrison, 1989). Therefore, the idea of replacing the wave function with the charge density was proposed in the so-called Thomas-Fermi (TFA) approach to electronic theory (Teller, 1962). Although the TFA failed to produce any quantitatively impressive results, the basic idea - using the electron density turned out to be very promising and was expanded in density functional theory (DFT) formulated by Hohenberg and Kohn (Hohenberg and Kohn,1964, Kohn and Sham, 1965). However, also in this case the direct application of the variational procedure becomes impossible and one works around the problem with the help of the Kohn-Sham (KS) scheme - maping the many-particle problem onto a system of non-interacting particles with the same electron density $n(r)$.

The model presented in (Görling, 1994) and used in this article returns to the standard formulation of quantum mechanics, however considering the non-local character of the potential in the Schrödinger equation. As a result, the electron interaction with crystal lattice corresponds to the potential

where:

$$
V(\boldsymbol{r})=\sum_{i=1}^{N} V\left(\boldsymbol{r}-\boldsymbol{r}_{\boldsymbol{i}}\right)
$$

$V\left(\boldsymbol{r}-\boldsymbol{r}_{\boldsymbol{i}}\right)=-\frac{z e^{2}}{\left|\boldsymbol{r}-\boldsymbol{r}_{\boldsymbol{i}}\right|}$ means simple Coulomb potential between electron and ion positioned in point $\boldsymbol{r}_{\boldsymbol{i}}$.

As for the wave function, we require that it be defined in the volume element $\Delta V_{i}$ around each ion located at point $\boldsymbol{r}_{i}$. The value of the function at any point can be presented in the form of:

where:

$$
\Psi(\boldsymbol{r})=\sum_{i=1}^{N} \chi\left(\boldsymbol{r}-\boldsymbol{r}_{i}\right) \Psi\left(\boldsymbol{r}_{\boldsymbol{i}}\right)
$$

$\chi\left(\boldsymbol{r}-\boldsymbol{r}_{i}\right)$ means expansion coefficients.

Consequently, taking into account equations (1) and (2) the effect of the potential operator interaction on the wave function takes form:

$$
\hat{V} \Psi(\boldsymbol{r})=\sum_{i} V\left(\boldsymbol{r}-\boldsymbol{r}_{\boldsymbol{i}}\right) \sum_{j} \Psi\left(\boldsymbol{r}_{\boldsymbol{j}}\right) \chi\left(\boldsymbol{r}-\boldsymbol{r}_{j}\right)
$$

and can be presented in the form of $N$-dimensional matrix (Chrzanowski, 2013). We can limit ourselves just to the diagonal elements of the matrix, because it may be easily proved that the other elements disappear at conditions similar to those known as Born Karman conditions (Harrison, 1989).

Choosing the function $\chi\left(\boldsymbol{r}-\boldsymbol{r}_{i}\right)$ (according to Bloch theorem) in form of:

$$
\chi\left(r-r_{i}\right)=\exp \left(-k_{s}\left|r-r_{i}\right|\right)
$$

We can transform the stationary Schrödinger equation into the Fourier domain. Next using the theorem of convolution (Kohn and Sham, 1965) and taking the boundary conditions as in (Chrzanowski, 2013)

$$
E(k=0)=\varepsilon_{i}
$$

where: 
$\varepsilon_{i}$ means the first ionization potential, we get an analytical equation for electron energy in real metal:

$$
E(\boldsymbol{k})=\frac{k^{2} \hbar^{2}}{2 m}-\frac{4 e^{2} n \pi}{k^{2}+\frac{4 e^{2} n \pi}{\varepsilon_{i}}}
$$

The equation above as well as the rest equations of the presented work are expressed in CGS units. Having equation (6), we can sum up after the all allowed and occupied states and thus obtain the density energy $\varepsilon$ of the system of $N$ interacting electrons.

$$
\varepsilon=\frac{E}{V}=\int_{0}^{K}\left(\frac{k^{2} \hbar^{2}}{2 m}-\frac{4 e^{2} n \pi}{k^{2}+\frac{4 e^{2} n \pi}{\varepsilon_{i}}}\right) \frac{8 k^{2}}{\sigma^{3} \pi^{2}} \mathrm{~d} k
$$

Here $K$ is the value of the wave vector for the highest occupied electron energy state and corresponds to the Fermi radius $k_{F}$ in the free electrons theory (FET) (Ashcroft and Mermin, 1976, Harrison, 1989). The coefficient $\sigma$ appears as a result of the typical transition from the sum to the integral and is related to the volume of a single cell in the $k$ space. For the free electrons theory (Kittel, 1996) $\Omega_{0}=\frac{(2 \pi)^{3}}{V}$, so $\sigma=2$. It is obvious that including the electron interaction with the crystal lattice increases the degeneration of the electron states and consequently $\sigma<2$. (for the same reasons $K<k_{F}$ ). Calculation of the precise value of $\sigma$ and $K$ for given metal will be an important point in the further part of the work. Final equation for energy density we get after integration Eq.(7):

$$
\varepsilon=-\frac{4 K\left(40 e^{2} m n \pi-K^{4} \hbar^{2}\right)}{5 m \pi^{2} \sigma^{3}}+\frac{64 e^{3} n^{3 / 2} \operatorname{Arc} \operatorname{Cot}\left[\frac{2 e \sqrt{n} \sqrt{\pi}}{K \sqrt{\varepsilon_{i}}}\right]}{\sqrt{\pi} \sigma^{3} \sqrt{\varepsilon_{i}}}
$$

\section{CALCULATION OF THE BULK MODULUS OF PURE METALS}

Knowing the total energy of the system of interacting electrons (8), we can use Gibbs ensemble and calculate the chemical potential for any metal:

$$
\mu=\left(\frac{\partial E}{\partial N}\right)_{V, S}=\frac{1}{V}\left(\frac{\partial E}{\partial n}\right)_{V, S}=\left(\frac{\partial \varepsilon}{\partial n}\right)_{V, S}
$$

In consequence we get:

$$
\mu=-\frac{32 e^{2} K}{\pi \sigma^{3}}-\frac{64 e^{4} n}{K \sigma^{3}\left(1+\frac{4 e^{2} n \pi}{K^{2} \varepsilon_{i}}\right) \varepsilon_{i}}+\frac{96 e^{3} \sqrt{n} \operatorname{ArcCot}\left[\frac{2 e \sqrt{n} \sqrt{\pi}}{K \sqrt{\varepsilon_{i}}}\right]}{\sqrt{\pi} \sigma^{3} \sqrt{\varepsilon_{i}}}
$$

Equation (8) also allows us to determine the bulk modulus, namely:

$$
B=n^{2} \frac{\partial^{2} \varepsilon}{\partial n^{2}}
$$

Thus, taking the second derivative after $n$ we obtain:

$$
B=\frac{48 e^{3} n^{3 / 2} \operatorname{Arc} \operatorname{Cot}\left[\frac{2 e \sqrt{n} \sqrt{\pi}}{K \sqrt{\varepsilon_{i}}}\right]}{\sqrt{\pi} \sigma^{3} \sqrt{\varepsilon_{i}}}-\frac{384 e^{6} K n^{3} \pi}{\sigma^{3}\left(4 e^{2} n \pi+K^{2} \varepsilon_{i}\right)^{2}}-\frac{160 e^{4} K^{3} n^{2} \varepsilon_{i}}{\sigma^{3}\left(4 e^{2} n \pi+K^{2} \varepsilon_{i}\right)^{2}}
$$

This is the equation that allows to calculate directly the bulk modulus for any metal much more accurately than, for instance the Hartree-Fock theory (Ashcroft and Mermin, 1976, Harrison, 1989), even taking $\sigma=2$ and $K=k_{F}$ as in the free electrons theory. The difference between the experimental value and the value calculated directly from the equation (12) for most metals does not exceed a several percent. Therefore, we can assume that the equation above is correct and that the discrepancies with the experiment come from approximations FET with respect to $k_{F}$ and $\sigma$. With this in mind, we can use this equation to determine the precise values both parameters $K$ and $\sigma$, so important from the point of view of quantum physics.

Because for $T=0 K, \mu=E(K)$, we can compare the right-hand sides of equations (6) and (10). This relation with Eq. (12) (in which we assume that we know the experimental value $B$ ) gives a set of equations with two unknowns $K, \sigma$ which we can solve numerically. In this way, we can 
find interested us parameters for any metals. Table1 shows the precise values of the two parameters obtained in this manner for $\mathrm{Cu}, \mathrm{Zn}, \mathrm{Al}$ and $\mathrm{Ni}$.

Table 1.

The precise value of $\sigma$ and $\mathrm{K}$ for chosen metals.

\begin{tabular}{|c|c|c|c|c|c|}
\hline metal & $\boldsymbol{K} \mathbf{1 0}^{\mathbf{8}} \mathbf{c m}^{-1}$ & $\boldsymbol{k}_{\boldsymbol{F}} \mathbf{1 0}^{\mathbf{8}} \mathbf{c m}^{-1}$ & $\boldsymbol{\sigma}$ & $\boldsymbol{\sigma}$ FET & $\boldsymbol{B}$ GPa \\
\hline $\mathrm{Cu}$ & 0.94 & 1.71 & 0.70 & 2 & 140 \\
\hline $\mathrm{Zn}$ & 1.07 & 1.57 & 1.2 & 2 & 70 \\
\hline $\mathrm{Al}$ & 1.08 & 2.07 & 0.80 & 2 & 76 \\
\hline $\mathrm{Ni}$ & 1.23 & 2.53 & 0.72 & 2 & 181 \\
\hline
\end{tabular}

As can be seen from the table above for such values $\sigma$ and $K$ we obtain the exact value of the bulk modulus $B$ for $\mathrm{Cu}, \mathrm{Zn}, \mathrm{Al}$ and $\mathrm{Ni}$ respectively. Please note, that as we have previously expected, both values are significantly lower than those resulting from FET.

\section{BULK MODULUS OF COPPER ALLOYS}

As it results from the equation (12), the bulk modulus of metal depends on four parameters, the ionization energy $-\varepsilon_{i}$, which for each metal is uniquely defined, the electron density $n$, which can be easily calculated knowing the number of valence electrons, and $K, \sigma$ which we can designate as above. However, in the case of alloys, the problem becomes even more complicated, because in this case we must create a mathematical model according to which the parameters above change. Of course, this model must also assume a dependence on the percentage composition of the alloy $-\alpha$.

So let us assume that the changes of these parameters in the case of a two-component alloy describe the system of equations:

$$
\begin{gathered}
n=\frac{N_{A}\left(\alpha M_{2} Z_{1}+(1-\alpha) M_{1} Z_{2}\right) \rho_{1} \rho_{2}}{M_{1} M_{2}\left((1-\alpha) \rho_{1}+\alpha \rho_{2}\right)}, \\
\varepsilon_{i}=\left(\alpha \varepsilon_{11}+(1-\alpha) \varepsilon_{\mathrm{i} 2}\right), \\
\sigma=\left(\alpha \sigma_{1}+(1-\alpha) \sigma_{2}\right), \\
K=\left(\alpha K_{1}+(1-\alpha) K_{2}\right) .
\end{gathered}
$$

Here $Z, M, \rho$ mean - valence, atomic mas and density of given component. Substituting these relations above into the equation (12) we can determine the dependence of $B$ of any alloy of two metals on their percentage composition. The following graphs Fig.1, Fig. 2 and Fig. 3 show this dependence for typical copper alloys.

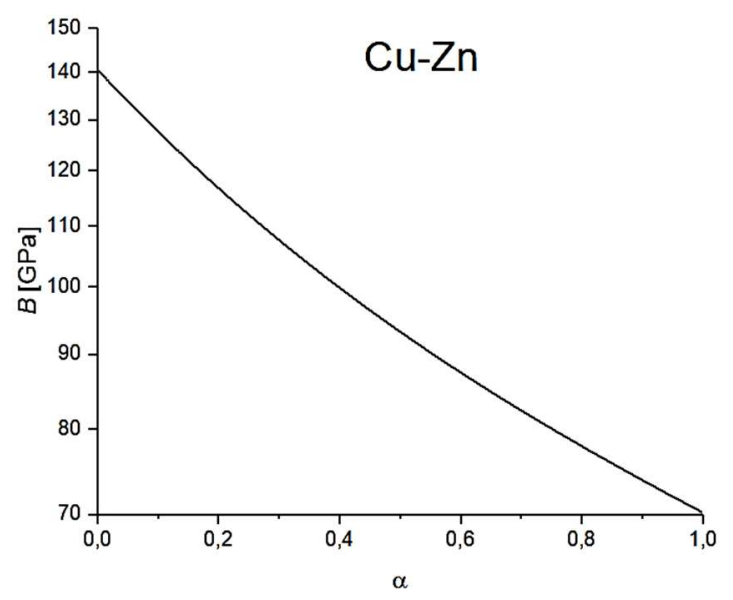

Fig.1. Dependence of the bulk modulus of $\mathrm{Cu}-\mathrm{Zn}$ on the percentage composition.

Note that, in spite of the relatively simple model, the dependence of $B$ on $\alpha$ is not linear, which is particularly evident in the case of Cu-Ni alloy Fig. 3. Just as you would expect (in any case) for $\alpha=0$, we obtain the bulk modulus of $\mathrm{Cu}$ whereas for $\alpha=1$ the bulk modulus of given metal. 


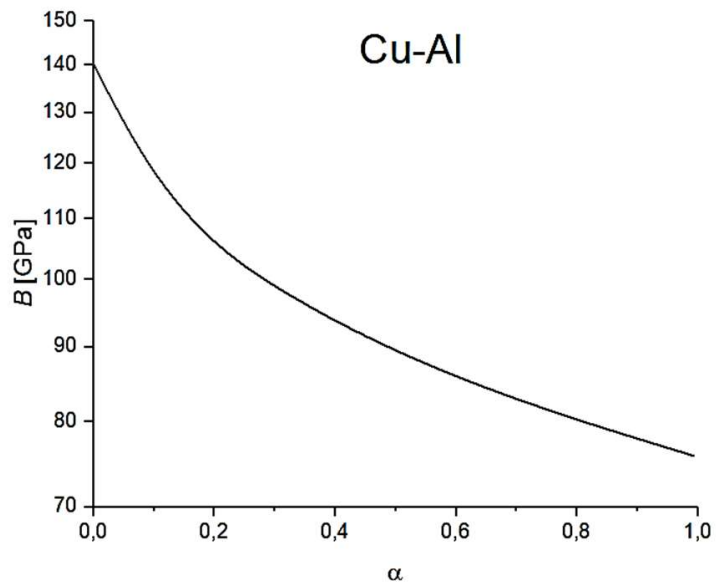

Fig. 2. Dependence of the bulk modulus of $\mathrm{Cu}-\mathrm{Al}$ on the percentage composition.

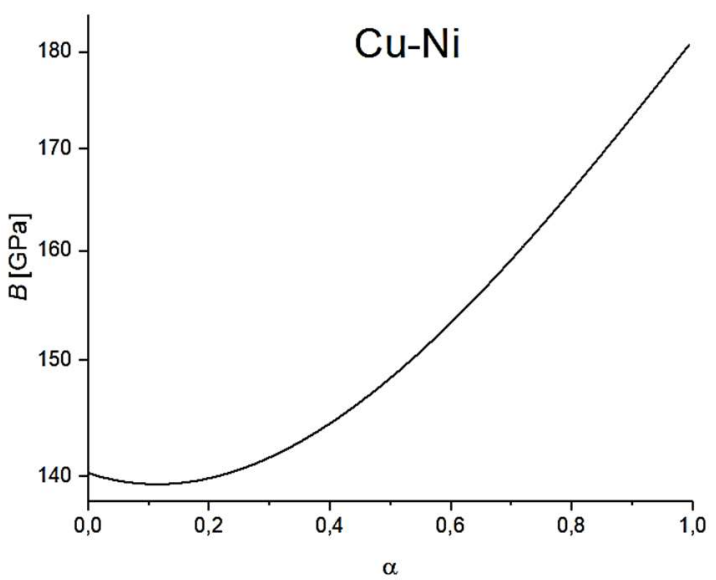

Fig. 3. Dependence of the bulk modulus of $\mathrm{Cu}-\mathrm{Ni}$ on the percentage composition.

\section{CONCLUSIONS}

The analytical equations presented in this paper permit to calculate with great accuracy the bulk modulus of any metal. Moreover, that equation can be used to calculate values of bulk modulus for alloys of different metals. However, in this case, we need to adopt an appropriate model for the changes in a pure metal energy structure caused by admixture (parameters $K$ and $\sigma$ ). $A$ simple model has been proposed, leading to significant differences that can be perceived during measurements. Because copper alloys are of particular interest, corresponding numerical calculations were made for three typical alloys: $\mathrm{Cu}-\mathrm{Zn}, \mathrm{Cu}-\mathrm{Al}, \mathrm{Cu}-\mathrm{Ni}$. Of course, the presented equations can be applied also to multi-component alloys. In perspective, it may be interesting to analyze the relationship of $B(T)$ for both copper and its alloys (Ledbetter, 1981) due to phase changes occurring in the case of $\mathrm{Cu}$ under the influence of temperature. Since now there are no experimental results referring (Zhang et al., 2008) to bulk modulus of metal alloys; and in particular, copper alloys, so the presented article might be an interesting starting point for research in this area, a research enabling a better understanding not only the mechanical properties of alloys but also the mechanism of electron interactions on a microscopic level. Therefore, the authors hope that the presented work will be an inspiration for research in this field, research important both for physicists and material science engineers.

\section{ACKNOWLEDGEMENTS}

This research outcome has been achieved under the research project No 1/S/KFiCh/14 financed from a subsidy of the Ministry of Science and Higher Education for statutory activities. 


\section{REFERENCES}

Ashcroft, N.W. and Mermin, N.D. (1976). Solid State Physics. New York: Holt, Rinehart and Winston.

Chrzanowski, J. (2013). Some Corrections to the Thomas-Fermi Theory. Chin. Phys. B 22(8), pp. 087101.

Görling, A. and Levy, M. (1994). Exact Kohn-Sham scheme based on perturbation theory. Phys. Rev. A 50(1), pp. 196-204.

Harrison W. A. (1989). Electronic Structure and the Properties of Solids. New York: Dover.

Hohenberg, P. and Kohn W. (1964). Inhomogeneous Electron Gas. Phys. Rev., 136(3B), pp. B864-B871.

Kittel, C. (1996). Introduction to Solid State Physics. New York: John Wiley \& Sons, Inc.

Kohn, W. and Sham, L.J. (1965). Self-Consistent Equations Including Exchange and Correlation Effects. Phys. Rev., 140(4A), pp. A1133-A1138.

Ledbetter, H.M. (1981). Elastic Constants of Polycrystalline Copper at Low Temperatures. Relationship to Single-Crystal Elastic Constants. Phys. Stat. Sol. (a), 66(2), pp. 477-484.

Powell, C. and Webster, P. (2012) Copper Alloys for Marine Environments. Copper Development Association Publication.

Teller E. (1962). On the stability of molecules in the Thomas-Fermi theory. Rev. Mod. Phys. 34(4), pp. 627-631.

Zhang, Y., Camilleri, J. A. and Zhu S. (2008), Mechanical Properties of Superelastic Cu-Al-Be Wires at Cold Temperatures for the Seismic Protection of Bridges. Smart Materials and Structures, 17(2), pp. 025008.

Date of submission of the article to the Editor: 04/2018

Date of acceptance of the article by the Editor: 07/2018 